\title{
Mathematical Model for Secretion of Leptin, Growth Hormone and Luteinizing Hormone
}

\author{
Geetha. $\mathrm{T}^{*}$ and Sangeetha.B** \\ *Asst professor of mathematics K.N Govt Arts college for women, Thanjavur. Tamilnadu, Southindia. \\ **Lecturer of mathematics, BDU college Orathanadu, Thanjavur, Tamilnadu, Southindia
}

\begin{abstract}
Estimation of $r$ when $y$ is censored at $x$ was applied to altered secretion of leptin, growth hormone and luteinizing hormone after $84 \mathrm{~h}$ of sustained physical exertion super imposed on caloric and sleep restriction an estimator based on a maximum likelihood estimates(MLEs) for reliability when stress is censored at strength. We assume that $X$ is a random strength of a component subjected to a random stress $(Y)$ and reliability of a component is given by $R=P[Y<X]$, where $(X, Y)$ follows the bivariate exponential (BVE) model of Marshall-Olkin.
\end{abstract}

Keywords: Bivariate exponential model, Maximum likelihood estimate, GH,LH.

\section{Introduction}

The pulsatile release of growth hormone (GH) and luteinizing hormone ( $\mathrm{LH})$ from the anterior pituitary gland is integral for signaling secretion of insulin-like growth factor (IGF)-I and testosterone, respectively. This study examined the hypothesis that $84 \mathrm{~h}$ of sustained physical exertion with caloric and sleep restriction alters the secretion of GH and LH. The pulsatile release of GH and LH from the anterior pituitary gland is integral for the trophic effects that these hormones exert on IGF-I and testosterone, respectively.The manner in which anterior pituitary hormones are released and delivered to target tissues is more biologically significant than the overall mean hormonal concentrations was well illustrated by Isgaard et al. , who demonstrated that GH administered in a pulsatile manner elicited greater increases in IGF-I than GH administered in a continuous manner. Because of the episodic release pattern of $\mathrm{GH}$ and $\mathrm{LH}$, multiple time-point measures are necessary to fully characterize the trophic effects these hormones may have. Although alterations in IGF-I and testosterone are known to be mediated by fluctuations in $\mathrm{GH}$ and $\mathrm{LH}$, few studies have concomitantly measured these hormones, especially in healthy subjects exposed to multiple physiological stressors. Military operational scenarios represent a unique paradigm in which to study endocrine responses to metabolic stress. Soldiers, analogous to emergency rescue personnel and ultra endurance athletes are often required to perform heavy sustained physical exertion in the face of nutritional and sleep deprivation. Prior work on military populations has shown that continued and prolonged exposure to such physiological strain can lead to a suppression of circulating anabolic growth factors, losses of lean body mass, increased susceptibility to disease and infections, and diminished physical and cognitive performance capabilities.Fourteen healthy male soldiers volunteered to participate in the study. Three injuries and one illness prevented four of these test subjects from completing the study. The data from the remaining 10volunteers [22 $\mathrm{yr}$ (SD 3), $183 \mathrm{~cm}$ (SD 7), $87 \mathrm{~kg}$ (SD 8)] are presented in this paper. The experiment was approved by the appropriate Institutional Scientific Review and Human Use Review Committees.The subjects were briefed on the study procedures, and written consent was obtained before study participation. Mean GH concentration was significantly increased after military stress compared with the control condition. The increase in mean GH concentration was accompanied by an increase in the following secretion parameters: area under bursts, amplitude of bursts, basal secretion rate, overnight basal secretion, overnight pulsatile secretion, and total overnight secretion. GH half-life, number of bursts, interval between bursts, and amplitude of largest burst were similar between the control and military operational stress conditions

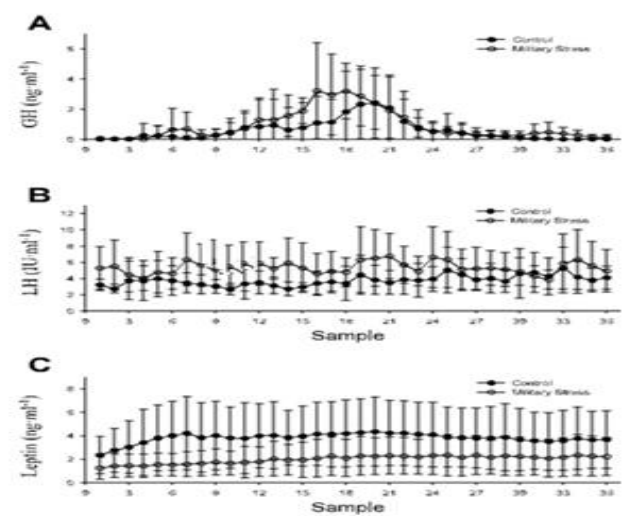


Mean concentration of all subjects over the 12-h sampling period.Samples were taken every 20 min for a total of 36 blood draws as represented on the $x$-axis. Main effect mean concentrations of GH $(A), \mathrm{LH}(B)$, and leptin $(C)$ after the military stress condition are statistically different $(P \leq 0.05)$ from control for all hormones

The fact that short-term (4 days) military operational stress can have striking effects on the neuroendocrine system raises questions for future studies concerning the potential adverse impact of long-term military operational stress on soldier physiology and the subsequent health and fitness of our military personnel.This study used a rigorous serial blood sampling scheme and subsequent time-series deconvolution analyses to assess GH and LH secretion patterns. These data expand on prior research that relied on one timepoint blood samples to glean informationon the endocrine responses to stress that are subject to episodic and circadian release patterns. The physiological significance of studying the pulsatile release of these hormones lies in the fact that the episodic release pattern is thought to be integral in eliciting optimal agonist function at the cellular level.The salient finding emerging from this study was an amplification of GH and LH secretion with a concomitant decline in IGF-I and testosterone. Because GH and LH are known to positively regulate IGF-I and testosterone, these data imply that the physiological strain experienced in this study induced a certain degree of peripheral resistance.

Despite enhanced release of LH, testosterone concentrations decreased. During the 12-h overnight sampling period, total and free testosterone concentrations were 24 and $30 \%$ lower after the military stress condition, respectively. By considering the circadian rhythm of testosterone, our study has extended these findings by demonstrating that testosterone concentrations are decreased and remain depressed overnight for a 12-h period following the conclusion of a multiple-day military stress condition. compared with younger men, older men tend to possess greater LH secretion, which is hypothesized to represent a biological attempt to maintain normal testosterone concentrations.Endurance-trained athletes have also shown decreased testosterone and increased LH concentrations. The literature with regard to acute exercise stress has been equivocal. Thus, although LH pulsatility is slowly restored in women, there is evidence to suggest that gonadotropin releasing hormone neurons may rapidly respond to caloric intake. Leptin concentrations declined from 3.8 to $2.0 \mathrm{ng} / \mathrm{ml}$ after military stress. These declines were coincident with marked reductions in fat mass and increased concentrations of circulating free fatty acids (33\%). This magnitude decrease for leptin is consistent with the $44 \%$ decrease reported by Maccario et al.After $36 \mathrm{~h}$ of fasting, but less than the $66 \%$ decline observed by Gomez-Merino et al. fter a 5-day military combat course. These changes are reflective of substantial lipolytic activity. Also of interest is the fact that the overall main effect mean for glucose concentrations was similar between the control and military stress condition, suggesting that glucose homeostasis was well defended in this paradigm. Although not measured in the present study, other studies have reported robust changes in cortisol after military stress, and it is also probable that both cortisol and catecholamine elevations played a contribution in maintaining glucose concentrations. The fact that the absolute losses in fat-free mass and fat mass were similar suggests that amino acid fuel partially contributed to gluconeogenesis. Converging lines of evidence in sheep, rat, monkey, and human models suggest that leptin acts as a peripheral signal to mediate metabolic regulation of $\mathrm{GH}$ and $\mathrm{LH}$ secretion from the anterior pituitary gland. Leptin appears to prevent the fastinginduced suppression of pulsatile release of LH secretion. In a recent study that evaluated the effects of differing doses of exogenously administered Leptin in men during $73 \mathrm{~h}$ of fasting and employing 24-h blood sampling, Chan et al. demonstrated a role for leptin in normalizing starvation-induced neuroendocrine changes, notably the hypothalamic- pituitary-gonadal axis. Chan et al. hypothesized that a threshold leptin level between 0.5 and 2.0 $\mathrm{ng} / \mathrm{ml}$ may be necessary for LH secretion. Although glucose concentrations have also been speculated to act as a peripheral signal relaying information concerning nutritional status and within the context of the dramatic hormonal changes observed, it is remarkable that glucosehomeostasis was well maintained.

\section{Mathematical Model}

Let $\left\{\left(x_{i}, y_{i}\right)\right\}$ be an i.i.d.random sample of size $\mathrm{n}$ from BVE of Marshall Olkin(1967). The strength and stress associated with the $\mathrm{i}$-th pair of the Sample is

$$
\begin{aligned}
\left(X_{i}, Y_{i}\right) & =\left(x_{i}, x_{i}\right) \text { if } x_{i} \leq y_{i} \\
& =\left(x_{i}, x_{i}\right) \text { if } x_{i}>y_{i}
\end{aligned}
$$

The likelihood of the sample of size $\mathrm{n}$ is

$$
L=\left(\prod_{i=1}^{n_{1}} f_{1}\right)\left(\prod_{i=1}^{n_{2}} f_{2}\right)
$$

Where

$$
f_{1}=\lim _{\delta x_{i} \rightarrow 0} \frac{P\left[X \in N\left(x_{i}\right), Y \geq X\right.}{\delta x_{i}}
$$


$=\left(\lambda_{1}+\lambda_{3}\right) \exp \left\{-\lambda x_{i}\right\}$,

$$
\begin{gathered}
f_{2}=\lim _{\delta x_{i} \rightarrow 0, \delta y_{i} \rightarrow 0} \frac{P\left[X \in N\left(x_{i}\right), Y \in N\left(y_{i}\right), Y<X\right.}{\delta x_{i} \delta y_{i}} \\
=\lambda_{2}\left(\lambda_{1}+\lambda_{3}\right) \exp \left\{-\lambda_{2} y_{i}-\left(\lambda_{1}+\lambda_{3}\right) x_{i}\right\},
\end{gathered}
$$

$\mathrm{n}_{1}\left(\right.$ or $\left.\mathrm{n}_{2}\right)$ is the number of observations with $\mathrm{x}_{\mathrm{i}} \leq \mathrm{y}_{\mathrm{i}}\left(\right.$ or $\left.\mathrm{x}_{\mathrm{i}}>\mathrm{y}_{\mathrm{i}}\right)$ in the sample of size $\mathrm{n}=\mathrm{n}_{1}+\mathrm{n}_{2}$.

$\mathrm{X} \in \mathrm{N}\left(x_{\mathrm{i}}\right)$ [ or $\mathrm{Y} \in \mathrm{N}\left(\mathrm{y}_{\mathrm{i}}\right)$ ] denotes $\mathrm{X}($ or $\mathrm{Y})$ fall in the neighborhood of $x_{i}\left(\right.$ or $\left.y_{i}\right)$ i.e., $x_{i}<\mathrm{X}<\mathrm{x}_{\mathrm{i}}+\delta_{\mathrm{xi}}$, for a small increment $\delta$ around $\mathrm{x}_{\mathrm{i}}\left(\right.$ or $\mathrm{y}_{\mathrm{i}}<\mathrm{Y}<\mathrm{y}_{\mathrm{i}}+\delta \mathrm{y}_{\mathrm{i}}$, for a small increment $\delta$ around $\left.\mathrm{y}_{\mathrm{i}}\right)$. The expected values of $\mathrm{n}_{1}$ and $\mathrm{n}_{2}$ are $\mathrm{E}\left(\mathrm{n}_{1}\right)=\left(\lambda_{1}+\lambda_{3}\right) / \lambda$ and $\mathrm{E}\left(\mathrm{n}_{2}\right)=\lambda_{2} / \lambda$

Letting $\gamma=\lambda_{1}+\lambda_{3}$, the above like hood can be written as

$$
\mathrm{L}=\gamma^{n} \lambda_{2}^{n_{2}} \exp \left\{-\gamma \sum_{i=1}^{n} x_{i}-\lambda_{2} \sum_{i=1}^{n} \min \left(x_{i}, y_{i}\right)\right\}
$$

It is observed from the above expression that we have complete observations on $\mathrm{X}$ and $\operatorname{Min}(\mathrm{X}, \mathrm{Y})$ in this censored scheme. It is a very important feature in the case of stress censored at strength. The like hood equations with respect to the parameters $\left(\lambda_{2}, \gamma\right)$ are

$$
\begin{array}{ll}
\mathrm{n}_{2} / \lambda_{2} & =\sum_{i=1}^{n} \min \left(x_{i}, y_{i}\right) \\
\mathrm{n} / \gamma & =\sum_{i=1}^{n} x_{i}
\end{array}
$$

The MLEs of $\left(\lambda_{2}, \gamma\right)$ are

$$
\hat{\lambda}_{2}=n_{2} / \sum_{i=1}^{n} \operatorname{Min}\left(x_{i}, y_{i}\right) \text { and } \hat{\gamma}=n / \sum_{i=1}^{n} \operatorname{Min} x_{i}
$$

One important observation here is that we have closed form expressions for the MLEs of $\left(\lambda_{2} \gamma\right)$ in this censored scheme but in the complete sample scheme the $M^{2} E_{S}$ of the parameters do not have explicit expressions and can be obtained by either by Newton-Raphson procedure or Fisher's method of scoring. [See Hangal and Kale(1991)].

The asymptotic variance-covariance matrix of $\left(\left(\lambda_{2}{ }^{\prime} \mathrm{Y}\right)\right.$ is

$$
\mathrm{V}\left(\hat{\lambda}_{2}\right)=\lambda_{2} \lambda / \mathrm{n}, \mathrm{V}(\hat{\gamma})=\gamma^{2} / \mathrm{n} \text { and } \operatorname{Cov}\left(\hat{\lambda}_{2}, \hat{\gamma}\right)=0 \text {. }
$$

Now the MLE of $\mathrm{R}$ is given by $\hat{R}_{1}=\hat{\lambda}_{2} /\left(\hat{\lambda}_{2}+\hat{\gamma}\right)$ and its asymptotic distributions is $\mathrm{AN}[\mathrm{R}, \mathrm{R}(1-$ $\mathrm{R})^{2}(1+\mathrm{R}) / \mathrm{n}$ ]. Jana (1994) obtained the estimate of $\mathrm{R}$ based on the complete sample on $\mathrm{X}$ and $\operatorname{Min}(\mathrm{X}, \mathrm{Y})$; i.e., $\hat{R}_{2}=1-\sum_{i=1}^{n} \min \left(x_{i}, y_{i}\right) / \sum_{i=1}^{n} x_{i}$, which is $\mathrm{AN}\left[\mathrm{R}, 2 \mathrm{R}(1-\mathrm{R})^{2} / \mathrm{n}\right]$. One can also have an estimator based on count $\mathrm{n}_{2}$ which is binomial(n, $\left.\mathrm{R}\right)$. The natural estimateor of $\mathrm{R}$ is $\hat{R}_{3}=n_{2} / n$ which is $\mathrm{AN}[\mathrm{R}, \mathrm{R}(1-\mathrm{R}) / \mathrm{n}]$.

The asymptotic relative efficiency (ARE) of the estimator $\hat{R}_{i}$ to the estimator $\hat{R}_{j}$ is given by

$$
\operatorname{ARE}\left(\hat{R}_{i}, \hat{R}_{j}\right)=\operatorname{var}\left(\hat{R}_{j}\right) / \operatorname{var}\left(\hat{R}_{i}\right) \quad i \neq j=1,2,3
$$

The estimator $\hat{R}_{1}$ is more efficient as compared to $\hat{R}_{2}$ and $\hat{R}_{3}$. The estimator $\hat{R}_{2}$ is more than efficient than the estimator $\hat{R}_{3}$ when $\mathrm{R}>.5, \hat{R}_{3}$ is more efficient than $\hat{R}_{2}$ when $\mathrm{R}<.5$, and both are equally efficient when $\mathrm{R}=.5$.

\begin{tabular}{|l|l|l|}
\hline \multicolumn{1}{|c|}{$\mathrm{X}$} & \multicolumn{1}{|c|}{$\mathrm{F}_{1}$} & \multicolumn{1}{c|}{$\mathrm{F}_{2}$} \\
\hline 0.2 & 0.1802 & 0.1120 \\
\hline 0.4 & 0.0483 & 0.0503 \\
\hline 0.6 & 0.0129 & 0.0237 \\
\hline 0.8 & 0.0035 & 0.0114 \\
\hline 1 & 0.0009 & 0.0055 \\
\hline 1.2 & 0.0002 & 0.0026 \\
\hline 1.4 & 0.0001 & 0.0013 \\
\hline 1.6 & 0.000018 & 0.0036 \\
\hline 1.8 & 0.000005 & 0.0017 \\
\hline 2 & 0.000001 & 0.0027 \\
\hline
\end{tabular}


Likelihood of leptin $=0$

Reliability of leptin $=0.8978$

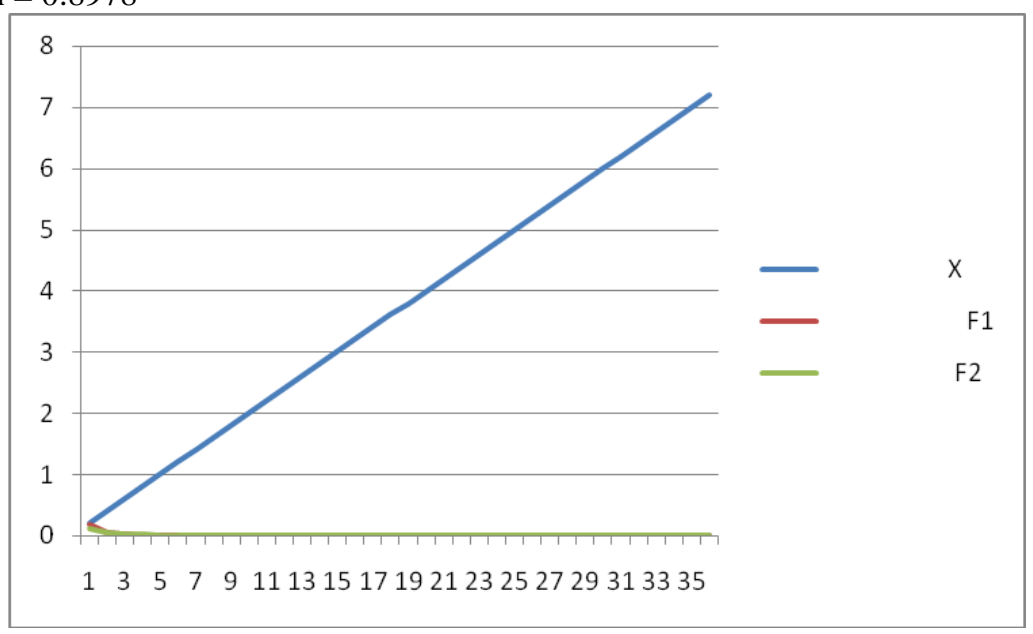

\section{Conclusion}

That short-term military operational stress can exert marked effects in neuroendocrine secretion patterns raises important questions concerning the potential adverse effects on longer-term military operational stress on underlying physiology and metabolism and the health status of military personnel and also found likelihood of sample for 36 size of leptin.

\section{References}

[1]. Castellani JW, Stulz DA, Degroot DW, Blanchard LA, Cadarette BS, Nindl BC, and Montain SJ. 84 hours of sustained operations alter thermoregulation during cold exposure. Med Sci Sports Exerc 35: 175-181, 2003.

[2]. Friedl KE, Moore RJ, Martinez-Lopez LE, Vogel JA, Askew EW, Marchitelli LJ, Hoyt RW, and Gordon CC. Lower limit of body fat in healthy men. $J$ Appl Physiol 77: 933-940, 1994.

[3]. Friedl KE, Moore RJ, Hoyt RW, Marchitelli LJ, Martinez-Lopez LE, and Askew W. Endocrine markers of semistarvation in healthy lean men in a multistressor environment. J Appl Physiol 88: 1820-1830, 2000

[4]. Hanagal, D.D.(1995). Testing reliability in a bivariate exponentialstress-strength model. Journal of the Indian StatisticalAssociation, $33,41-45$.

[5]. Johnson ML, Virostko A, Veldhuis JD, and Evans WS. Deconvolution analysis as a hormone pulse-detection algorithm. Methods Enzymol 384: 40-54, 2004.

[6]. Kramer TR, Moore RJ, RL Shippee, KE Friedl, L Martinez-Lopez, MM Chan, and Askew EW. Effects of food restriction in military training on T-lymphocyte responses. Int J Sports Med 18: 84-90, 1997.

[7]. Marshall, A.W. and Olkin, I.(1967). A multivariate exponential distribution. Journal of the American Statistical Association, 62, 3044.

[8]. Nindl BC, Friedl KE, Frykman PN, Marchitelli LJ, Shippee RL, and Patton JF. Physical performance and metabolic recovery among lean, healthy men following a prolonged energy deficit. Int J Sports Med 18: 317-324, 1997.

[9]. Veldhuis JD, Anderson SM, Shah N, Bray M, Vick T, Gentili A, Mulligan T, Johnson ML, Weltman A, Evans WS, and Iranmanesh A. Neurophysiological regulation and target-tissue impact of the pulsatile mode of growth hormone secretion in the human. Growth Horm IGF Res 11, Suppl A: 25-37, 2001

[10]. Veldhuis JD. Issues in quantifying pulsatile neurohormone release. In:Methods in Neuroendocrinology. Baton Rouge, FL: CRC, 1998, p. 181-203 